\title{
Outcomes of total mesopancreas excision in patients with carcinoma head of pancreas with pancreaticoduodenectomy
}

\author{
Sai SAING WAN KHAM, Lin TUN THEIN, Tin TIN MAR*
}

HBP Surgery Department, Yangon Specialty Hospital, University of Medicine 1, Yangon, Myanmar

Introduction: Retro pancreatic invasion is a major concern in pancreatic head carcinoma. Posterior resection margin has been recognized as risk factor for disease recurrence and hence patient survival. In pancreaticoduodenectomy, with total mesopancreas excision, more posterior resection margin is achieved and hence locoregional recurrence rate is lowered and survival is prolonged.

Methods: This study was a hospital-based, prospective, interventional study starting from November 2018 to June 2020, at Hepatobiliary and Pancreatic Surgery Department, Yangon Specialty Hospital. There were 27 patients with resectable carcinoma head of pancreas patients in this study. In all patients, pancreaticoduonectomy with total mesopancreas excision was done and the outcomes such as posterior resection margin clearance rate, mesopancreas lymph node involvement by tumor, locoregional recurrence rate and survival rate were analyzed.

Results: Posterior resection margin clearance was achieved in $70.37 \%$. On the average 4 mesopancreas lymph nodes were removed and mesopancreas lymph node involvement by tumor was seen in $37 \%$ of patients with high positivity ratio; $100 \%$ positivity ratio in half of mesopancreas lymph node involved patients. Postoperative complications was seen in $30 \%$ of study population including perioperative mortality. POPF was seen in $7.4 \%$, PPH was seen in $14 \%$ and the perioperative mortality rate was $14.8 \%$. No locoregional recurrence was found, but one case of liver metastasis developed at postoperative 6 th month. This study's half year overall survival rate was $85.2 \%$ and half year recurrence free survival rate was $84.6 \%$.

Conclusions: With total mesopancreas excision, posterior resection margin clearance was achieved in $70 \%$ with mesopancreas lymph node involvement in $37 \%$. 